\title{
Combustion graphology used to improve emulsions of water-in-heavy fuel oil
}

\author{
by Victor V. Ghia and A. Colibaba-Evulet
}

\author{
Institute for Thermal Engines, Bucharest, 246 Pacii Blvd., Sector 6, Romania
}

\begin{abstract}
Combustion graphology uses the infrared and luminous radiation of the flame and cenosphere of a burning droplet of heavy fuel oil in order to carry out scientific research with technical industrial applications. The rational combustion of the optimal water-heavy fuel oil emulsions in industrial power furnaces determines several advantages of which the most important is the de-pollution of the environment. Graphological testing for water-heavy fuel oil emulsion droplets is performed on a simulator and the result of the experiments is presented for two situations: when the effect of secondary atomisation is partially present and when the secondary atomisation is total, the whole droplet exploding. Experimental results are presented for samples processed in laboratory and in an industrial emulsifying installation.
\end{abstract}

\section{Generalities}

The combustion of mixtures of water $(20 \%-30 \%)$ and heavy fuel oil, especially for attaining the reduction in smoke and soot emissions is a well-known method. Recently, this method was considerably improved in order to avoid the increase both of sulphur corrosive action and of the specific fuel consumption. First of all, it has been replaced the use of mixture of water and heavy fuel oil with emulsions, the water content in the emulsion being drastically reduced. Several beneficial advantages may be obtained by burning homogeneous emulsions of waterheavy fuel oil, with the water content limited to $3-6 \%$ [1], [2].

Due to the existence of a large number of various types of heavy fuel oil and emulsifying installations and in order to optimise the useful effects it is necessary to improve experimentally the quality of the water-heavy fuel oil emulsions. The emulsifying installations have adequate specific functioning rates; out of the range of these rates, the installation produces low quality emulsions, which are inappropriate to use. This work firstly gives explanations on what is the mechanism of the combustion of water-heavy fuel oil emulsions, the conclusions being based on experiments. The specific aspects of the droplet combustion graphology are herein set and a rapid and precise method for establishing the quality of waterheavy fuel oil emulsions is proposed. The validity of this method was verified in industrial and laboratory experiments. Some of these characteristic results are presented together with the applications of the combustion graphology method, which offers the possibility of fast and accurate processing of very economical activities in different fields.

\section{Secondary atomisation}

The meaning of the appearance of advantageous effects when using water-heavy fuel oil emulsions is related to the so-called secondary atomisation. If the water is finely dispersed into the fuel oil, the water droplets diameter is of the order of about 2-5 $\mu \mathrm{m}$. During the primary atomisation process, these small water droplets will be incorporated into the oil droplets, the latter ones having a magnitude order of $20-150 \mu \mathrm{m}$. When the droplets enter the incandescent combustion chamber, they are heated up and a sudden vaporisation of the water droplets occurs, leading to a further atomisation of the oil droplets into smaller ones. The result is the secondary atomisation, produced by micro-explosions, which appear as a consequence of the important increase of the vapour pressure caused by the release of fine water droplets from the emulsion.

As a conclusion, many constituents are released from the combustible mass of the fuel droplet. These components need a lower time to burn. The important decrease of the 
combustion time is mainly the result of the increase of the combustible mass surface area in contact with the atmospheric oxygen. Obtaining the double atomisation, primary and secondary, constitutes the physical explanation to the beneficial development of the combustion of the water-liquid fuel emulsions.

\section{Graphological characteristics of heavy fuel oils}

Combustion graphology of heavy fuel oils is defined as the scientific branch concerned with the graphical transposition of these fuels' combustion processes, establishing the ignitioncombustion characteristics including the laws that govern their changes, depending on the combustion conditions and the chemical structure of the heavy fuel oils [3]. The characteristic quantities of combustion graphology of heavy fuel oils result from the interpretation of the combustion oscillograms of some droplets, experimentally obtained under the form of a curve represented in a rectangular system of co-ordinate axes. The $\tau$ time variation is represented in the abscissa and the I radiation intensity variation of the burning droplet of heavy fuel oil is represented in the ordinate, this variation being transformed into voltage by means of a photoelectric cell with amplification, which receives the infrared light signals. Figure 1 shows the combustion oscillogram for a droplet of heavy fuel oil, which ignites and bums in the symmetry centre of a hot cylindrical combustion chamber attached to the simulator [4].

The main characteristic quantities used by the combustion graphology of heavy fuel oils are:

- self-ignition delay $\tau_{i}$;

- combustion time of the volatile matters $\tau_{\mathrm{v}}$;

- cenosphere ignition delay $\Delta \tau$, when it is distinctively marked on the oscillogram;

- combustion time of the cenosphere $\tau_{\mathrm{c}}$;

- total time of combustion :

$$
\tau_{\mathrm{a}}=\tau_{\mathrm{v}}+\tau_{\mathrm{c}}
$$

- droplet existence time:

$$
\tau_{\mathrm{a}}=\tau_{\mathrm{i}}+\tau_{\mathrm{a}}
$$

- maximum radiation intensity obtained at the burning of the volatile matters $I_{\mathrm{v}}^{\mathrm{m}}$;

- maximum radiation intensity obtained at the burning of the cenosphere $I_{\mathrm{c}}^{\mathrm{m}}$;

- flame radiated energy, transformed by the photocell into electric energy, due to the buming of the volatile matters:

$$
E_{\mathrm{v}}=k \int_{0}^{\tau v} f(\tau) \mathrm{d} \tau
$$

where $k$ is a specific constant depending on the simulator type;

- energy radiated by the burning cenosphere, transformed by the photocell into electric energy:

$$
E_{\mathrm{c}}=k \int_{0}^{\tau \kappa-\Delta \tau} F(\tau) \mathrm{d} \tau
$$

In order to make a more systematic distinction of the heavy fuel oils from the ignition, combustion and radiation points of view, and considering at the same time all the above mentioned characteristics, the following quantities were defined:

- combustion simplex :

- radiation index:

$$
S=\tau_{\mathrm{i}} \cdot \tau_{\mathrm{v}}^{-{ }^{-1}}
$$

$$
B=E_{\mathrm{v}} \cdot\left(E_{\mathrm{v}}+E_{\mathrm{c}}\right)^{-1}
$$

- complex index of ignition and combustion:

$$
G=\tau_{v}\left(\tau_{i}+\tau_{c}\right)^{-1}
$$

The experimental research showed that the quality improvement of the heavy fuel oils is specified by the increase of the quantities $B, G$ and the decrease of the simplex $S$. The selfignition delay time $\tau_{i}$ is the time elapsed from the droplet insertion inside the combustion chamber until the droplet ignition, which occurs at the same time with the appearance of the flame, marked on the abscissa axis $O \tau$ by the point $a$. The volatile matters burning time $\tau_{v}$ is 
the time elapsed from the droplet ignition moment (point a) until the flame extinction (point $b$ ). Cenosphere ignition delay time $\Delta \tau$, when existing, is the time elapsed from the moment of flame extinction until the visible ignition of the cenosphere (point $c$ ), which is marked by the appearance of its radiation in the visible spectrum. It should be mentioned that the chemical reactions of the cenosphere ignition appear before the moment of time marked by the point $c$.

In many heavy fuel oils having $\Delta \tau=0$, the cenosphere burning is following the thin line curve of figure 1. In this case, the minimum of the curves $I_{y}=f(\tau)$ and $I_{c}=F(\tau)$, corresponding to the abscissa of point $b^{\prime \prime}$, will specify the beginning of the cenosphere burning time. The $\Delta \tau \geq$ 0 quantity mainly depends on the combustion conditions, the $D_{0}$ diameter, and the quality of the heavy fuel oil. The cenosphere buming time $\tau_{c}$ was defined as the period of time elapsed between the moment of the extinction of the flame produced by the volatile matters' combustion (point $b$ or point $b^{\prime \prime}$ ) and the moment of burning cenosphere extinction (point $d$ ).

The method of combustion testing of the fuel oils consists of the controlled ignition and burning of a fuel oil droplet, under standard conditions. The standard conditions are mainly specified by:

- the geometry of the combustion chamber;

- the initial temperature $T_{f 0}$ and the pressure approximately equal to the atmospheric pressure inside the combustion chamber;

- the environmental temperature $T_{\mathrm{a}}$ and the heavy fuel oil temperature $T_{\mathrm{c}}$;

- the system of supporting and inserting the droplet inside the combustion chamber;

- the air flow conditions around the droplet, mainly characterised by the value of the Reynolds number, Re;

- the mean initial diameter $D_{0}$ of the combusted droplet.

The determination of the ignition and combustion graphological characteristics of heavy fuel oils needed the construction of a specific testing rig also called simulator, consisting of the following main components [4]:

- the electric heated cylindrical combustion chamber, inside a metallic casing cooled by the water circulation generated by a pump;

- the device for the propulsion of the droplets in-suspension, on the extremity of a quartz filament which is pointing towards the interior of the combustion chamber;

- the optical equipment for centring, processing, focusing (on the photocell) and viewing the image of a burning droplet ;

- the electronic equipment consisting in a device for signalling the insertion of the droplet inside the combustion chamber, two photovoltaic transducers, amplifier and processor for the electric current signals, filter and parasitic radiation's compensatory devices.

- two electric heaters including annexes, the first for heating the ceramic wall of the combustion chamber, made of four silica rods and the second one for pre-heating the air, the latter having an automatic temperature regulating device;

- an electronic computer for experimental data acquisition, processing, displaying and storing, all resuits from the combustion oscillograms;

- a syringe divider with specialised microscope and ocular-interpolating device for the precise droplet calibration at given dimensions.

\section{The characteristic experimental results}

From the numerous experimental results obtained so far, we will present firstly those completed in the laboratory, using emulsions of water and heavy fuel oil type A specification RMF 25, ISO 8217/1987. The liquid whirling homogenisation system was used for emulsifying the water in heavy fuel oil. The tests on the emulsion droplets have been performed in the simulator for the following water contents: $0 \%, 5 \%, 10 \%, 15 \%$ and $20 \%$, under the same standard conditions. For example, the oscillogram for the combustion of an A-type heavy fuel oil droplet with $D_{0}=2.2 \mathrm{~mm}, T_{\mathrm{a}}=291 \mathrm{~K}$ and $T_{\mathrm{c}}=330 \mathrm{~K}$, ignited at $T_{\mathrm{fo}}=973 \mathrm{~K}$ and $\operatorname{Re}=140$, generates the following mean values: 


$$
\tau_{\mathrm{i}}=540 \mathrm{~ms}, \tau_{\mathrm{v}}=2430 \mathrm{~ms}, \tau_{\mathrm{c}}=8310 \mathrm{~ms}, S=3.4198, B=0.6532, G=0.2509 .
$$

The combustion oscillogram for a droplet of water in A-type heavy fuel oil emulsion with a water content of $w=10 \%$, with $D_{0}=2.2 \mathrm{~mm}, T_{\mathrm{a}}=291 \mathrm{~K}$ and $T_{\mathrm{c}}=330 \mathrm{~K}$, ignited at $T_{\mathrm{fo}}=973 \mathrm{~K}$ and $R e=140$, generates the following mean values:

$$
\tau_{\mathrm{i}}=568 \mathrm{~ms}, \tau_{\mathrm{v}}=1104 \mathrm{~ms}, \tau_{\mathrm{c}}=2976 \mathrm{~ms}, \mathrm{~S}=2.6957, B=0.8890, G=0.3115 \text {. }
$$

By analysing the results obtained from the combustion oscillograms and for a variation of the water content from $w=0$ to $20 \%$, the change in the characteristic quantities results as follows:

- the combustion time for the cenosphere, $\tau_{\mathrm{c}}$ varies from $8310 \mathrm{~ms}$ to $2300 \mathrm{~ms}$;

- the net specific energy $P_{\mathrm{ci}}$ varies from $41.4 \mathrm{MJ} / \mathrm{kg}$ to $32.75 \mathrm{MJ} / \mathrm{kg}$;

- the self-ignition delay time $\tau_{\mathrm{i}}$ varies from $540 \mathrm{~ms}$ to $650 \mathrm{~ms}$;

- the combustion time for the volatile matter, $\tau_{c}$, varies from $2430 \mathrm{~ms}$ to $1000 \mathrm{~ms}$.

When the water content of the emulsion increases to a maximum value of $10 \%$ to a small, approximately linear, increase of the self-ignition delay $\tau_{i}$ it corresponds a sensitive and rapid drop for $\tau_{c}$ time.

The experiments presented above correspond to the case of less intensive secondary atomisation effect due to a poor quality of the emulsifying process. There is an important number of water droplets with a diameter exceeding the mean diameter value of $6 \mu \mathrm{m}$. However, in such a case, in order to obtain a proper de-pollution for the combustion, it is not recommended, for economic reasons, to increase the water content beyond the limit of $6 \%$. The industrial research performed on special centrifugal pumps as emulsifying installations proved that particular functioning rates may be found in order to improve the secondary atomisation for higher beneficial effects.

The heavy fuel oil was of the B-type, corresponding to RMG 35 specification, ISO 8217/1987. The combustion was experimented in similar standard conditions. From the combustion oscillogram for a droplet of water - B type heavy fuel oil emulsion $(w=3 \%)$, with $D_{0}$ $=1.9 \mathrm{~mm}, T_{\mathrm{a}}=293 \mathrm{~K}$ and $T_{\mathrm{c}}=310 \mathrm{~K}$, which was ignited at $T_{\mathrm{fo}}=1023 \mathrm{~K}, \mathrm{Re}=135$, one may observe the following:

$$
\tau_{\mathrm{i}}=260 \mathrm{~ms}, \tau_{\mathrm{v}}=1070 \mathrm{~ms}, \tau_{\mathrm{c}}=1150 \mathrm{~ms} ;
$$

- the maximum increase of the temperature $T_{f}$ inside the combustion chamber is $\Delta T_{f}=73 \mathrm{~K}$; the slide of the $T_{f}=\Psi(\tau)$ curve being the consequence of the thermal inertia of the temperature transducer, which impedes overlaying the $I_{v}=f(\tau)$ curve;

- the $I_{v}=f(\tau)$ curve presents a gap in the central part, due to the sensitive water content which produces obstructing vapours as a result of the volatile matter combustion;

- the energy radiated at the combustion of the volatile matter, $E_{\mathrm{v}}$ and represented to scale by the area below the $I_{v}=f(\tau)$ curve, is sensitively greater than the energy radiated at the combustion of the cenosphere which is represented by the area below the $I_{c}=F(\tau)$.

We appreciate that for the droplets of the considered emulsion, the effect of secondary atomisation, respectively the explosive combustion, appears only to some extent. This deficiency may be corrected by using the emulsion of water $(w=6 \%)$ and B-type heavy fuel oil (figure 2), where for a droplet of $D_{0}=1.9 \mathrm{~mm}, T_{\mathrm{a}}=293 \mathrm{~K}$ and $T_{\mathrm{c}}=310 \mathrm{~K}$, ignited at $T_{\mathrm{fo}}=$ $1023 \mathrm{~K}, \mathrm{Re}=135$, one may observe the following:

$$
\tau_{\mathrm{i}}=285 \mathrm{~ms}, \tau_{\mathrm{v}}=727 \mathrm{~ms}, \tau_{\mathrm{c}}=734 \mathrm{~ms} \text {; }
$$

- $\Delta T_{\mathrm{f}}=45 \mathrm{~K}$, much less than the preceding case, due to the decrease of the flame temperature and a shorter flame;

- the $T_{\mathrm{f}}=\Psi(\tau)$ curve presents a acute growth when the flame appears; 
- the $\tau_{\mathrm{v}}$ and $\tau_{\mathrm{c}}$ time parameters are sensitively smaller, due to the intensified appearance of the micro-explosions if compared to the preceding case and finally, the droplet wholly explosion;

- $E_{\mathrm{c}} \ll E_{\mathrm{v}}$ due to the same reason, which means $B \rightarrow 1$.

This represents the optimal rate of functioning of the water-heavy fuel oil emulsifier; the resulting emulsion was tested, and it has confirmed to reach the best results when burnt in the boiler [5].

\section{Conclusions}

The optimal quality of the water-heavy fuel oil emulsion is determined expensively and in a difficult way, indirectly, by determining the diameter and number of the water droplets contained in the emulsion with an electronic microscope or, directly, by analysing the beneficial effects of the industrial combustion of the emulsion. The proposed method eliminates the above mentioned disadvantages by using the combustion graphology of the liquid fuels. The method consists in testing a water-heavy fuel oil emulsion droplet at ignition-combustion and by adequately interpreting the results, instantly displayed on the computer's screen.

When analysing by this method the combustion of a water-heavy fuel oil emulsion droplet, two possible distinct cases may appear:

- the first case, when $\tau_{c}, E_{c}$ and $I_{c}{ }^{m}$ are sensitively decreasing due to the faster combustion of the cenosphere, the effect of secondary atomisation being partially present;

the second case, when the secondary atomisation is total, the whole droplet exploding during, or after, the volatile matters' combustion, thus $\tau_{\mathrm{c}}=0, E_{\mathrm{c}}=0$ and $I_{\mathrm{c}}^{\mathrm{m}}=0$, with $S=0$ and $B=1$. This second case is determining the optimal quality of the emulsion that, when burned, may lead to the achievement of maximal beneficial effects. The classification into the first or the second category depends on:

- (principally) the quality of the emulsion, which is given by the emulsifying installation type used and its rate;

- the disposal of the heavy fuel oil to be emulsified;

- the thermal conditions inside the furnace where the droplets' combustion occurs; these conditions are favourable to intensifying the combustion at higher relative temperatures, that may be obtained when using low amounts of water in the emulsion.

For the above mentioned cases, the combustion time of volatile matters, $\tau_{v}$ is decreasing due to the obstructing influence of water vapours on the combustion process. The sudden increase of temperature $T_{\mathrm{f}}$ inside the combustion chamber of the simulator when burning the volatile matter is also a characteristic of high quality water-heavy fuel oil emulsions. The graphological characteristics $\tau_{c}, E_{c}, I_{c}{ }^{m}$ and the shape of the $T_{f}=\Psi(\tau)$ curve, obtained from the combustion oscillogram, are the main criteria for establishing the quality of an emulsion. The application of the graphology method, together with its embodying improved installations offer the possibility to accurately carry out very economical activities in various fields as results from [4].

\section{REFERENCES}

[1] SJOGREN (A). - Buming of water in oil emulsion, Technological Institute, Copenhagen, Denmark, 1987, p. 297-305

[2] ABRIL (A.) - Emulsion d'eau, solution pour la bonne combustion du fuel lound en chaudières, Rev.Gen.Therm.Fr., 305, 1987, p. 334.

[3] GHIA (V.V.), Combustion Graphology of Heavy Fuel Oils, Energetica, seria A, 6, 1994, p. 268-274

[4] GHIA (V.V.) - Combustion Graphology of Intermediate Fuel Oils, Revue Roumain Science Techn.- Électrotechn. Et Énerg., 36,3, 1991, p. 379-396,

[5] GHIA (V.V.) and RADULESCU (M.) - New criteria for improving the water-heavy fuel oils by using the combustion graphology, "The XXII ${ }^{\text {nd }}$ National Conference for Termoenergetics", 7-9 December, Brasov, Romania, 1995. 


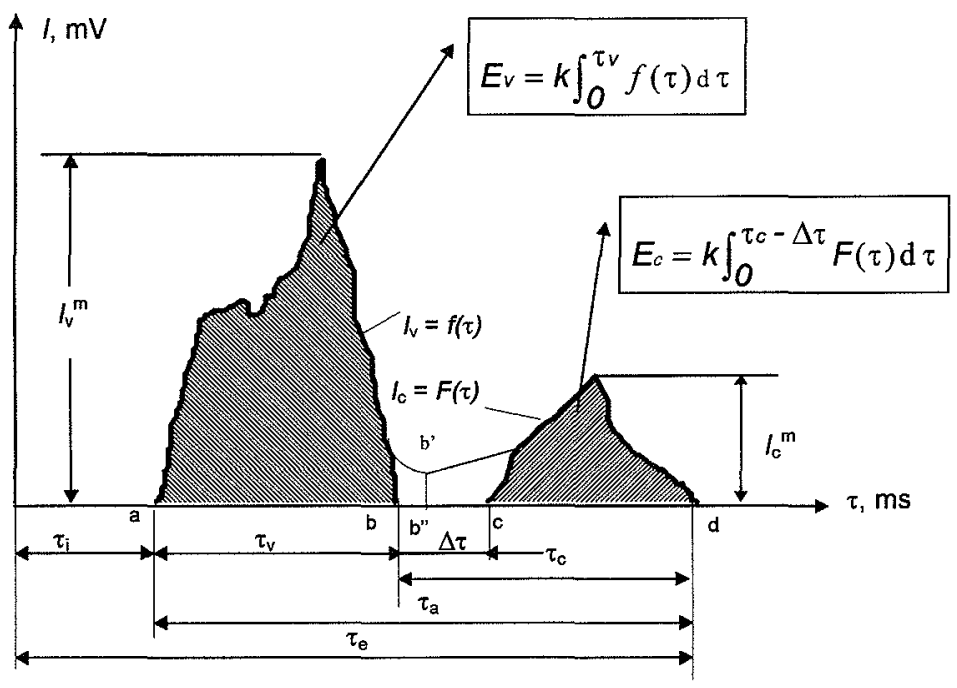

Fig. 1: Combustion oscillogram for a droplet of different qualities of heavy fuel oil

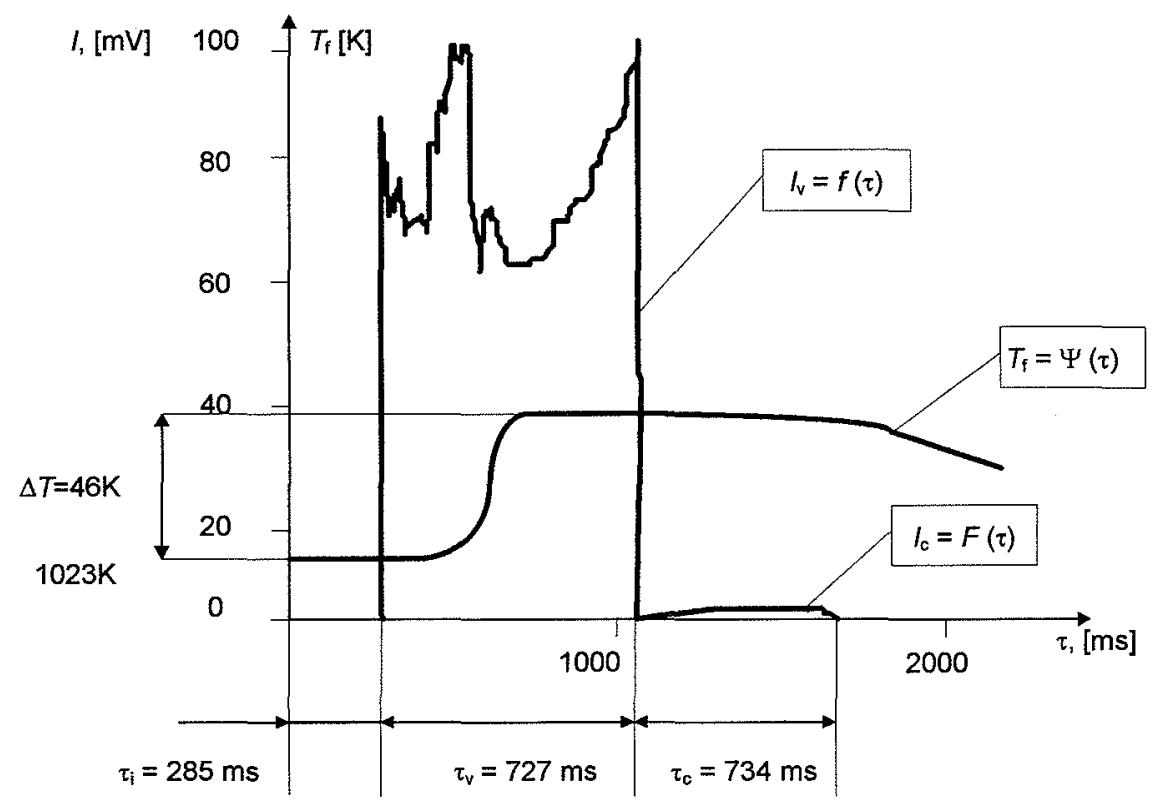

Fig. 2: Combustion oscillogram for a droplet of water-B type heavy fuel oil ( $w=6 \%$ ) with $D_{0}=1.9 \mathrm{~mm}$, ignited at $T_{\mathrm{fo}}=1023 \mathrm{~K}$ and $\mathrm{Re}=135$ 